CARDIOVASCULAR MEDICINE

\title{
Reduction of daily life ischaemia by aspirin in patients with angina: underlying link between thromboxane A2 and macrophage colony stimulating factor
}

\author{
I lkonomidis, F Andreotti, P Nihoyannopoulos
}

Heart 2004;90:389-393. doi: 10.1136/hrt.2003.015164

See end of article for authors' affiliations

\section{Correspondence to:} Dr lgnatios Ikonomidis, University of Athens, Department of Clinical Therapeutics, Alexandra Hospital, Perikleous 19, N Chalkidona, Athens 14343, Greece; ignoik@ otenet.gr

Accepted 30 July 2003

\begin{abstract}
Objectives: To evaluate whether aspirin reduces the incidence and frequency of daily life myocardial ischaemia in a cohort of patients with chronic stable coronary artery disease.

Setting: Tertiary referral centre.

Methods: 60 patients with chronic stable coronary artery disease underwent 48 hour Holter monitoring to assess the incidence and frequency of daily life myocardial ischaemia. Those with myocardial ischaemia $(40 / 60)$ entered a double blind, crossover trial of aspirin $(300 \mathrm{mg} /$ day for three weeks) versus placebo. After each treatment arm, 48 hour Holter monitoring was repeated and urinary thromboxane (Tx) B2, 11 dehydro-TxB2, plasma prothrombin fragment $\mathrm{F} 1+2$, macrophage colony stimulating factor (MCSF), and interleukin (IL)- 6 were measured.

Results: Aspirin reduced the total number and duration of ischaemic episodes from 339 to 251 and from 1765 to 1365 minutes, respectively ( $<0.01$ for both). TxB2 was also reduced from 0.2 to $0.1 \mathrm{ng} / \mathrm{mg}$ creatinine, 11-dehydro-TxB2 from 3.3 to $1.3 \mathrm{ng} / \mathrm{mg}$ creatinine, $\mathrm{Fl}+2$ from 1.5 to $1.2 \mathrm{nmol} / \mathrm{l}, \mathrm{MCSF}$ from 991 to $843 \mathrm{pg} / \mathrm{ml}$, and IL-6 from 3.5 to $2.9 \mathrm{pg} / \mathrm{ml}$ (p $<0.05$ for all). 11-Dehydro-TxB2 excretion with and without aspirin was related to MCSF concentrations $(p<0.01)$, and the percentage reduction of MCSF by aspirin was related to the reduction of 11-dehydro-TxB2 $(p<0.05)$ and the reduction of the ischaemic burden compared with placebo $(p<0.05)$.

Conclusions: In patients with daily life ischaemia, aspirin reduces the incidence and frequency of ischaemic episodes as well as the systemic concentrations of haemostatic/inflammatory markers. Aspirin may prevent transient coronary flow reductions through platelet, thrombin, and cytokine inhibition.
\end{abstract} revascularisation procedure (coronary artery bypass grafting or percutaneous transluminal coronary angioplasty) within

Abbreviations: $\mathrm{Fl}+2$, plasma prothrombin fragment $\mathrm{Fl}+2$; IL, interleukin; MCSF, macrophage colony stimulating factor; Tx,

the previous six months $(\mathrm{n}=5)$, clinical evidence of peripheral vascular disease $(n=1)$, or diabetes mellitus $(n=3)$. Twenty patients, included in a previous report, ${ }^{11}$ were also excluded from the current study because they did not have evidence of myocardial ischaemia on the baseline 48 Holter monitoring. Thus the final cohort consisted of 40 patients (36 men, four women; mean age 56 (6) years, range 38 to 68 ).

Written informed consent was obtained from all the patients. Other exclusion criteria not found in our cohort were evidence of cerebrovascular disease, renal or liver dysfunction, bleeding tendencies, malignancy, known inflammatory diseases, and allergy to aspirin.

\section{Study protocol}

Upon enrolment for Holter monitoring, all antiplatelet drugs were withdrawn for seven days. At the end of this period, antianginal treatment was gradually reduced over the next 48 hours to avoid rebound ischaemia and was then withheld for a further 72 hours, during which the 48 hour period of Holter monitoring was undertaken (using a Marquette 8000 laser system). During this five day period, antiplatelet treatment was again withheld. Thus patients were off any antiplatelet treatment for an overall period of 12 days. Sublingual nitrates were allowed should chest pain persist beyond three minutes, but only five of the 40 patients made use of them. None of the

thromboxane 
patients was unable to complete the study protocol because of withdrawal of aspirin or antianginal treatment.

Evidence of ischaemia on Holter monitoring was defined as ST depression of more than $0.1 \mathrm{mV}$, occurring $60 \mathrm{~ms}$ after the $\mathrm{J}$ point and lasting for more than one minute. The maximum and mean heart rates, heart rate at the onset of ST depression, maximum ST depression, and the presence or absence of symptoms were noted. In all 40 patients the quality of the 48 hour Holter recordings was optimal.

The study was approved by the Hammersmith Hospital's research ethics committee at a time when aspirin treatment in stable patients was not implemented in routine clinical practice. $^{12}$

\section{Randomisation to aspirin or placebo}

Aspirin and placebo were provided by the hospital pharmacy as identical capsules. Aspirin was given at a dose of $300 \mathrm{mg} /$ day because, at this dose or higher, studies have shown that it may inhibit shear induced platelet activation ${ }^{13}$ and thrombin generation, ${ }^{14}$ and exert an anti-inflammatory effect. ${ }^{15}$ Each treatment lasted three weeks to avoid any carry over effects of aspirin. Twenty of the 40 patients with Holter evidence of ischaemia were initially randomised to aspirin, and the other 20 to placebo. After three weeks, all 40 patients were crossed over to the alternative treatment for another three weeks. Forty eight hours before the end of each three week phase, 48 hour Holter monitoring was repeated and blood sampling and urine collections were done. Patients and physicians were blinded to the type of treatment assigned (aspirin or placebo); the code was broken after data analysis had been completed.

\section{Blood and urine samples}

Forty eight hours before the end of each three week treatment period, morning blood samples were drawn on two consecutive days, and two 24 hour urine collections were taken for the measurement of the following factors: urinary thromboxane (Tx)A2 metabolites (TxB2 and 11-dehydroTxB2), as indices of platelet activation'; plasma prothrombin fragment $\mathrm{F} 1+2$, as an index of thrombin generation ${ }^{16}$; and interleukin (IL)-6 and macrophage colony stimulating factor (MCSF), as indices of inflammatory activity. ${ }^{11}$ Aliquots of plasma and urine were stored at $-70^{\circ} \mathrm{C}$ and analysed in batches within a year of sampling.

\section{Laboratory assays}

Urinary TxB2 and 11-dehydro-TxB2 were measured by enzyme linked immunoassays (Cascade Biochem, Neogen TM, Reading, UK; sensitivity $0.004 \mathrm{ng} / \mathrm{ml}$ for TxB2 and $0.01 \mathrm{ng} / \mathrm{ml}$ for 11 -dehydro-TxB2); values were corrected for the 24 hour urinary excretion of creatinine ( $\mathrm{ng} / \mathrm{mg}$ creatinine). Cross reaction with other prostanoids in the urine was $<0.25 \%$. The assays were in compliance with the US Food and Drug Administration's Good manufacturing practice regulations. Fl+2 concentrations were measured by enzyme linked immunoassay (Enzygnost Fl+2 micro, Behring, USA; sensitivity $0.04 \mathrm{nmol} / \mathrm{l}$ ). Plasma MCSF and IL- 6 were measured by enzyme linked immunoassays (R\&D System, Minneapolis, USA: human MCSF quantikinine, sensitivity $20 \mathrm{pg} / \mathrm{ml}$; and high sensitivity human IL-6 quantikinine, sensitivity $0.094 \mathrm{pg} / \mathrm{ml}$ ). The intra-assay coefficients of variation were less than $5 \%$ for all tests. The assays were done by personnel who were unaware of the clinical data.

\section{Statistical analysis}

The number of patients was determined so that the study would achieve a power more than $80 \%$ for detecting a reduction by aspirin of 2 (2.6) ischaemic episodes during Holter monitoring, a similar reduction having been observed after ticlopidine treatment. ${ }^{17}$ Differences within and among groups were analysed by the Wilcoxon signed rank test, the Mann-Whitney U test, or analysis of variance (KruskalWallis and Friedman test). Spearman's rank correlation was used to assess relations. Multiple relations were tested by stepwise regression analysis. Categorical variables were compared by contingency $\chi^{2}$ tests. Biochemical and ECG data are presented as medians and interquartile ranges unless otherwise stated. For each treatment phase, the mean of two consecutive blood and urine measurements was used. A two tailed probability value of $p<0.05$ was considered significant.

\section{RESULTS}

\section{Baseline characteristics}

The patients' main clinical characteristics are shown in table 1 . The right coronary artery was involved in 23 patients, the left anterior descending in 28, and the left circumflex in 23. All patients had an ejection fraction of more than $55 \%$ at left ventriculography. This study cohort of 40 patients with ischaemia during Holter monitoring was similar in age, sex, risk factor distribution, and mean and maximum heart rate at baseline to the 20 patients without ischaemia included in our previous report. ${ }^{11}$ Patients with ischaemia, however, had a higher prevalence of multivessel disease: 38/40 v 11/20 (94\% $v 55 \%, \mathrm{p}<0.05)$. None of the patients was admitted to hospital for a cardiac or any other reason during the study.

\section{Holter monitoring}

In the 40 patients included in the crossover trial with aspirin, 314 ischaemic episodes lasting in total 1678 minutes were recorded during baseline Holter monitoring. In these patients, mean heart rate at the onset of ischaemia during Holter

Table 1 Baseline clinical characteristics of the study population

\begin{tabular}{|c|c|}
\hline Variable & All patients $(n=40$ ) \\
\hline Age (years) & $56(6)$ \\
\hline $\operatorname{Sex}(M / F)$ & $36 / 4(90 \%)$ \\
\hline Cholesterol (mmol/l) & $6(1)$ \\
\hline Triglycerides (mmol/l) & $1.9(1.3)$ \\
\hline Hypertension (yes/no) & $15 / 25(37 \%)$ \\
\hline Parental CAD (yes/no) & $19 / 21(48 \%)$ \\
\hline Smoking (yes/no) & $23 / 17(57 \%)$ \\
\hline \multicolumn{2}{|l|}{ CCS anginal class } \\
\hline 1 & $16(40 \%)$ \\
\hline 2 & $19(48 \%)$ \\
\hline 3 & $5(12 \%)$ \\
\hline \multicolumn{2}{|l|}{ Diseased coronary arteries } \\
\hline 1 & $2(6 \%)$ \\
\hline 2 & $17(42 \%)$ \\
\hline 3 & $21(52 \%)$ \\
\hline Previous MI (yes/no) & $21 / 19(53 \%)$ \\
\hline \multicolumn{2}{|l|}{ Drugs* ${ }^{*}$} \\
\hline$\beta$ Blockers & $21 / 40(53 \%)$ \\
\hline Calcium channel blockers & $21 / 40(53 \%)$ \\
\hline Long acting nitrates & $10 / 40(25 \%)$ \\
\hline Lipid lowering & $6 / 40(15 \%)$ \\
\hline Statins & $3 / 40(8 \%)$ \\
\hline Fibrates & $3 / 40(8 \%)$ \\
\hline Antiplatelets & $14 / 40(35 \%)$ \\
\hline Diuretics & $6 / 40(15 \%)$ \\
\hline ACE inhibitors & $5 / 40(13 \%)$ \\
\hline Monotherapy† & $19 / 40(48 \%)$ \\
\hline GTN only & $7 / 40(18 \%)$ \\
\hline \multicolumn{2}{|c|}{$\begin{array}{l}\text { Values are mean (SD) or } n(\%) \text {. } \\
\text { *Medical treatment at inclusion. } \\
\text { †Patients on only one of the three classes of antianginal } \\
\text { drugs. } \\
\text { †Patients on only sublingual glyceryl trinitrate at inclusion. } \\
\text { ACE, angiotensin converting enzyme; CAD, coronary artery } \\
\text { disease; CCS, Canadian Cardiovascular Society; F, female; } \\
\text { GTN, glyceryl trinitrate; M, male; MI, myocardial infarction. }\end{array}$} \\
\hline
\end{tabular}


Table 2 Effects of aspirin on daily life ischaemia in patients with angina

\begin{tabular}{|c|c|c|c|c|}
\hline & \multicolumn{3}{|c|}{ Ischaemic episodes on 48 hour Holter monitoring } & \multirow[b]{2}{*}{ p Valuet } \\
\hline & Baseline* $(n=40)^{*}$ & Placebo $(n=40)$ & ASA $(n=40)$ & \\
\hline $\begin{array}{l}\text { Total number } \\
\text { Median number/patient } \\
\text { Total duration (min) } \\
\text { Median duration/patient (min) } \\
\text { Max ST depression (mV) } \ddagger \\
\text { Mean HR at onset (beats/min) }\end{array}$ & $\begin{array}{l}314 \\
8(4-12) \\
1678 \\
32(15-60) \\
0.14(0.12-0.20) \\
84(17)\end{array}$ & $\begin{array}{l}339 \\
7(3-11) \\
1765 \\
29(11-55) \\
0.13(0.12-0.17) \\
83(16)\end{array}$ & $\begin{array}{l}251 \\
5(1.3-8.8) \\
1305 \\
17(6.4-38) \\
0.16(0.14-0.21) \\
89(16)\end{array}$ & $\begin{array}{l}<0.01 \\
<0.01 \\
<0.01 \\
<0.01 \\
<0.01 \\
<0.05\end{array}$ \\
\hline \multicolumn{5}{|c|}{$\begin{array}{l}\text { Values are median (interquartile range) or mean (SD) unless stated. } \\
\text { *NS for all baseline } v \text { placebo comparisons. } \\
\text { †Values refer to ASA v placebo comparisons. } \\
\text { †Max ST depression, maximum ST depression } 60 \mathrm{~ms} \text { after the J point, lasting >1 min. } \\
\text { ASA, aspirin; HR, heart rate. }\end{array}$} \\
\hline
\end{tabular}

monitoring was significantly lower than that recorded during the previous exercise test (84 (17) $v 106$ (18) beats/min, $\mathrm{p}<0.01)$. Most ischaemic episodes on Holter monitoring occurred at heart rates of less than 90 beats/min (210 episodes, or $67 \%$ ). Maximum and mean heart rates and the number and duration of ischaemic episodes at baseline and at the end of the placebo phase of the aspirin trial were similar, indicating that the patients' level of activity and threshold for ischaemia remained stable throughout the study (table 2).

\section{Effects of aspirin on daily life ischaemia}

The total number of ischaemic episodes fell from 339 during placebo to 251 during aspirin, and their duration fell from 1765 to 1305 minutes $(\mathrm{p}<0.01$ for both comparisons, table 2). The episodes recorded during aspirin showed higher heart rates at their onset $(p<0.05)$ and greater ST depression $(p<0.01)$ compared with those recorded during placebo (table 2), indicating a reduction by aspirin in the number of episodes occurring at low heart rates.

\section{Effects of aspirin on haemostatic and inflammatory markers}

In the 40 patients with Holter evidence of ischaemia, treatment with aspirin for three weeks was associated with a median reduction in TxB2 of $45 \%$, in 11-dehydroTxB2 of $60 \%$, and in F1+2 of $20 \%$ compared with placebo $(\mathrm{p}<0.01$ for all comparisons) (table 3 ). As previously reported, ${ }^{11}$ MCSF fell by $19 \%$ and IL- 6 by $37 \%$ compared with placebo $(\mathrm{p}<0.05$ for both, table 3$)$. Urinary 11dehydro-TxB2 concentrations were related to those of plasma MCSF, both during placebo $(r=0.62, \mathrm{p}<0.01)$ (fig 1A) and after aspirin $(r=0.53, \mathrm{p}<0.01)$ (fig 1B). Moreover, the percentage reduction in MCSF during aspirin was related to the percentage reduction in 11-dehydro-TxB2 and in the duration of ischaemic episodes compared with placebo $(r=0.41$ and $r=0.43$, respectively; $\mathrm{p}<0.05)$. Patients who showed a more than $60 \%$ reduction in 11 -dehydro-TxB2 $(n=20)$ during aspirin compared with placebo were considered to be aspirin responders. They had lower MCSF
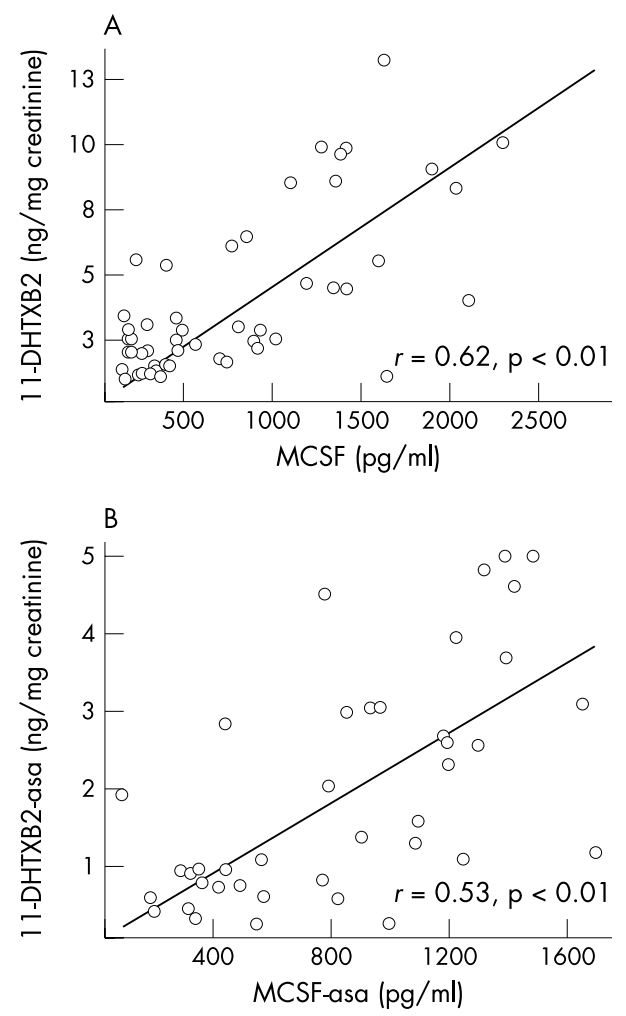

Figure 1 Relation between plasma macrophage colony stimulating factor (MCSF) concentrations and urinary excretion of 11 -dehydrothromboxane B2 (1 1-DHTxB2) (A) during placebo $(r=0.62$, $\mathrm{p}<0.01)$, and $(\mathrm{B})$ after aspirin administration $(r=0.53, \mathrm{p}<0.01)$.

values during both treatment phases than patients with $\leqslant 60 \%$ reduction in 11 -dehydro-TxB2, who were considered to be non-responders (placebo: 500 (370-930) pg/ml $v 1310$ (821-1612) pg/ml MCSF; aspirin: 552 (370-870) pg/ml $v$ 1136 (815-1395) pg/ml MCSF; p < 0.05 for all comparisons) (fig 2).

Table 3 Effects of aspirin on markers of haemostatic and inflammatory activity

\begin{tabular}{llll}
\hline & Placebo $(\mathbf{n}=40)$ & ASA $(\mathbf{n}=40)$ & p Value \\
\hline TxB2 (ng/mg creatinine) & $0.21(0.15-0.30)$ & $0.11(0.09-0.14)$ & $<0.01$ \\
$11-\mathrm{DHT}$ - 2 (ng/mg creatinine) & $3.3(2.3-5.4)$ & $1.3(0.8-3.1)$ & $<0.01$ \\
F1+2 (nmol/l) & $1.5(1.1-2.7)$ & $1.2(1.1-1.8)$ & $<0.01$ \\
$\mathrm{MCSF}(\mathrm{pg} / \mathrm{ml})$ & $991(459-1476)$ & $843(501-1357)$ & $<0.05$ \\
IL-6 $(\mathrm{pg} / \mathrm{ml})$ & $3.5(3.2-4.6)$ & $2.9(2.5-3.4)$ & $<0.05$ \\
\hline
\end{tabular}

Values are median (interquartile range)

$\mathrm{F} 1+2$, plasma prothrombin fragment $\mathrm{Fl}+2$; IL, interleukin; MCSF, macrophage colony stimulating factor; $\mathrm{TxB} 2$, thromboxane B2; 11-DHTxB2, 11-dehydro-thromboxane B2. 


\section{DISCUSSION}

We have previously shown that aspirin reduces proinflammatory cytokines in patients with chronic coronary artery disease. ${ }^{11}$ In the present study, we show that aspirin reduces myocardial ischaemia during daily life in patients with demonstrable ischaemia on 48 hour Holter monitoring, in proportion with the concomitant reduction in MCSF concentrations and in parallel with a reduction in platelet activation and thrombin generation.

\section{Daily life ischaemia and haemostatic/inflammatory markers}

MCSF induces monocyte/macrophage activation, ${ }^{18}$ plateletmonocyte adhesion and activation, ${ }^{19-21}$ and the release of platelet derived growth factor, thrombospondin, ${ }^{19} 22$ and vasoactive proteins, including $\operatorname{TxA} 2{ }^{8}$ endothelin, serotonin, leukotrienes, and oxygen radicals. ${ }^{182}$ Both MCSF and TxA2 are involved in vasomotion and in platelet and coagulation activation; therefore they may cause transient coronary flow reductions in patients with stable coronary disease through constriction of stenotic but pliable arteries ${ }^{26}{ }^{10}$ or through formation of temporary platelet aggregates and microthrombi that can spontaneously lyse or dislodge. ${ }^{2}{ }^{10}$ The lower heart rates at the onset of Holter monitored ischaemia compared with exercise induced ischaemia suggest that at least part of the ischaemic episodes recorded in our population was associated with transient reductions in coronary flow. Indeed, nearly $70 \%$ of the ischaemic episodes occurred at heart rates of less than 90 beats/min.

\section{Relation between TxA2 and MCSF and effects of aspirin on biochemical markers}

The significant relation between MCSF and TxA2 metabolites observed during the placebo phase is consistent with the ability of MCSF to promote platelet activation in vitro ${ }^{19-21}$ or with a proinflammatory effect of activated platelets. In the present study, TxA2 metabolites, prothrombin $\mathrm{F} 1+2$, and the measured inflammatory cytokines were significantly reduced by aspirin. While the reduction in TxA2 metabolites is easily explained by aspirin's irreversible block of platelet cyclooxygenase- 1,23 the reduced plasma concentrations of $\mathrm{F} 1+2$ may be a result of platelet inhibition ${ }^{13}{ }^{14}$ and of the reduced procoagulant actions of MCSF and IL- 6 by aspirin. ${ }^{1124-26}$ The significant relation between TxA2 biosynthesis and MCSF after aspirin administration suggests either aspirin resistant TxA2 production by platelets, ${ }^{27}$ leading to cytokine production, or stimulation of extra-platelet sources of TxA $2^{28}$ by the persistent MCSF production. Indeed, in the present study, aspirin responders had a twofold lower MCSF concentration than non-responders during placebo and aspirin treatment.

\section{Effects of aspirin on daily life ischaemia}

Aspirin given for three weeks to patients with Holter evidence of ischaemia produced a reduction in ischaemic episodes compared with placebo. MCSF and TxA2 metabolites were interrelated and were also significantly reduced by aspirin. A direct relation between the reduction of ischaemic episodes with aspirin and a parallel reduction in the systemic concentrations of haemostatic and inflammatory biochemical indices may be difficult to establish in the clinical setting, as the genesis of myocardial ischaemia is multifactorial. ${ }^{10}$ Remarkably, however, the percentage reduction in MCSF after aspirin treatment (compared with placebo) was independently related to both the percentage reduction in 11-dehydro-TxB2 and the percentage reduction in the duration of ischaemic episodes in the present study. Thus it is possible that aspirin reduced ischaemia through a combined reduction of the powerful vasoactive and haemostatic mediator $\mathrm{TxA}^{2}{ }^{923}$ and also of the proinflammatory and procoagulant cytokine MCSF. ${ }^{11}$ Interestingly, the ischaemic episodes that occurred during aspirin were at higher heart rates and greater ST depression (most probably secondary to increased myocardial demand) than those observed during the placebo phase, further suggesting that aspirin exerted a beneficial action on supply driven ischaemia. In agreement with this, Fox and colleagues found that treatment with ticlopidine reduced transient ischaemia accompanied by low heart rates in patients with both exertional and rest angina. ${ }^{17}$ On the other hand, we cannot exclude the possibility that aspirin reduced the number and duration of ischaemic episodes through other mechanisms, and that the reduction in haemostatic and inflammatory indices was merely secondary to the lessened ischaemic burden.

\section{Study limitations}

Cytokines and Fl+2 were measured in peripheral blood and TxA2 metabolites in the urine; therefore firm conclusions on the release of these factors within the coronary circulation cannot be drawn.

\section{Conclusions}

This prospective study in 40 patients with Holter evidence of myocardial ischaemia showed that aspirin treatment at a dose of $300 \mathrm{mg} /$ day reduced daily life ischaemia and haemostatic/inflammatory activity. These findings are clinically relevant, as daily life ischaemia ${ }^{29}$ and MCSF plasma concentrations ${ }^{30}$ are both known to predict adverse outcomes. Our data suggest a possible mechanism for the effect of aspirin on transient ischaemia-namely the combined
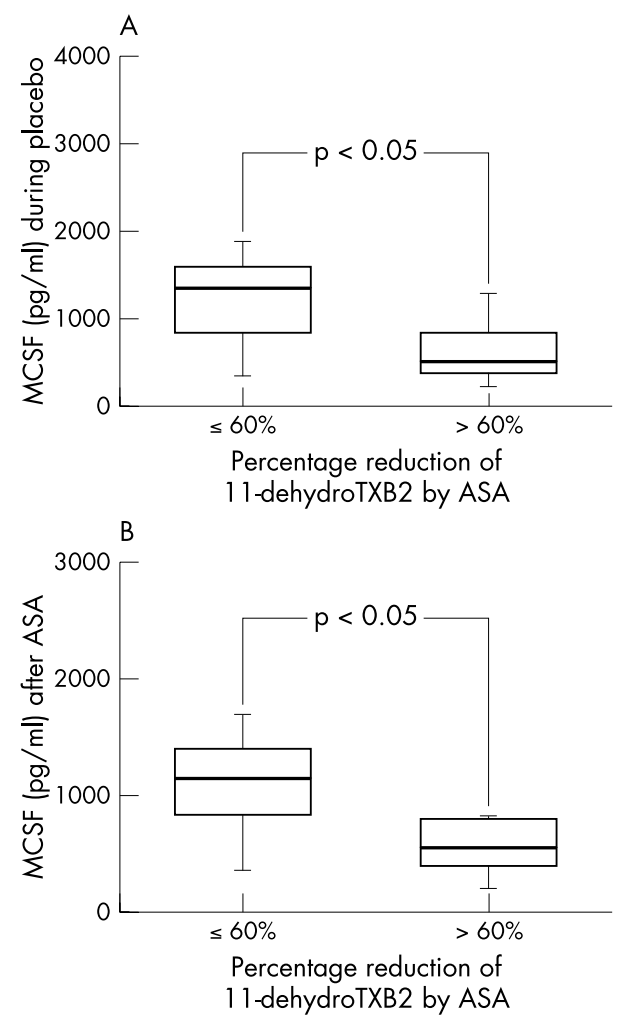

Figure 2 Plasma macrophage colony stimulating factor (MCSF) concentrations in patients with $\leqslant 60 \%$ and $>60 \%$ reduction in urinary excretion of 11-dehydro-thromboxane B2 (11-DHTxB2) by aspirin treatment, (A) during placebo $(p<0.05)$, and (B) after aspirin (ASA) administration $(p<0.05)$. Values are expressed as median with 25th and 75 th centiles by the box, with the largest and smallest values given by the lines at the ends of the box. 
inhibition of platelet activation, thrombin generation, and inflammatory cytokines. Indeed, the reduction in the concentrations of TxA2 metabolites and MCSF by aspirin was associated with the reduction in ischaemic burden. Whether lower doses of aspirin or other antiplatelet agents exert similar effects remains to be established. These results offer a mechanistic basis for the reported reduced rate of coronary events in patients with chronic stable angina treated with aspirin. ${ }^{12}$

\section{ACKNOWLEDGEMENTS}

This work was supported by Hammersmith Hospital grant No RC/ 259 .

\section{Authors' affiliations}

I lkonomidis, University of Athens, Department of Clinical Therapeutics, Alexandra Hospital, Athens, Greece

F Andreotti, The Institute of Cardiology, Catholic University Medical School, Rome, Italy

P Nihoyannopoulos, Cardiology Department, Imperial College School of Medicine, National Heart and Lung Institute, Hammersmith Hospital, London, UK

\section{REFERENCES}

1 Deanfield JE, Maseri A, Selwyn AP, et al. Myocardial ischaemia during daily life in patients with stable angina. Its relation to symptoms and heart rate changes. Lancet 1983;ii:753-8.

2 Folts JD, Crowell EB, Rowe CG. Platelet aggregation in partially obstructed vessels and its elimination with aspirin. Circulation 1976;54:365-70.

3 Andreotti F, Lanza GA, Sciahbasi A, et al. Low-grade exercise enhances platelet aggregability in patients with obstructive coronary disease independently of myocardial ischemia. Am J Cardiol 2001;87:16-20.

4 Davies MJ, Woolf N, Rowles PM, et al. Morphology of the endothelium over atherosclerotic plaques in human coronary arteries. Br Heart $J$ 1988;60:459-64.

5 Thompson SG, Kienast J, Pyke SD, et al. Hemostatic factors and the risk of myocardial infarction or sudden death in patients with angina pectoris. N Engl J Med 1995;332:635-41.

6 Hangartner JRW, Charleston AJ, Davies MJ, et al. Morphological characteristics of clinically significant coronary artery stenoses in stable angina. Br Heart J 1986;56:501-8.

7 Kaski JC, Tousoulis D, Haider AW, et al. Reactivity of eccentric and concentric stenoses in patients with chronic stable angina. J Am Coll Cardiol $1991 ; 17: 627-33$.

8 Ellis EF, Oelz O, Robert $\amalg$, et al. Coronary arterial smooth muscle cell contraction by a substance released from platelets: evidence that it is thromboxane A2. Science 1976;193:1135-8.

9 Patrono C, Davi G, Giabattoni G. Thromboxane biosynthesis and metabolism in relation to cardiovascular risk factors. Trends Cardiovasc Med 1992;2:15-20.

10 Fuster V, Badimon L, Badimon J, et al. The pathogenesis of coronary artery disease and the acute coronary syndromes (I). N Engl J Med 1992;326:242-50.
11 Ikonomidis I, Andreotti F, Economou E, et al. Increased proinflammatory cytokines in patients with chronic stable angina and their reduction by aspirin. Circulation 1999; 100:793-8.

12 Juul-Mollers S, Edvardsson N, Jahnmatz B, et al. Double-blind trial of aspirin in primary prevention of myocardial infarction in patients with stable chronic angina pectoris. The Swedish angina pectoris aspirin trial (SAPAT) group. Lancet 1992;340:1421-5.

13 Ratnatunga CP, Edmondson SF, Rees GM, et al. High-dose aspirin inhibits shear-induced platelet reaction involving thrombin generation. Circulation 1992;85: 1077-82.

14 Loew D, Vinazzer H. Dose-dependent influence of acetylsalicylic acid on platelet functions and plasmatic coagulation factors. Haemostasis 1976;5:239-49.

15 Ridker PM, Cushman M, Stampfer MJ, et al. Inflammation, aspirin and the risk of cardiovascular disease in apparently healthy men. N Engl J Med 1997;336:973-9.

16 Aronson DL, Stevan L, Ball AP, et al. Generation of the combined prothrombin peptide (F1.2) during clotting in blood plasma. J Cin Invest 1977:60:1410-18.

17 Fox KM, Jonathan A, Selwyn AP. Effects of platelet inhibition on myocardial ischaemia. Lancet 1982;2:727-30.

18 Roth P, Stanley ER. The biology of CSF-1 and its receptor. Current topics in microbiology and immunology. Berlin: Sonneg-Verlag, 1992;181:141-58.

19 Yesner LM, Huh HY, Pearse SF, et al. Regulation of monocyte CD36 and thrombospondin expression by soluble mediators. Arterioscler Thromb Vasc Biol 1996; 16:1019-25.

20 Silverstein RL, Nachman RL. Thrombospondin binds to monocytemacrophages and mediates platelet monocyte adhesion. J Clin Invest 1987;79:867-74.

21 Orlandi M, Bartolini G, Minghetti L, et al. Prostaglandin and thromboxane biosynthesis in isolated platelet-free monocytes. III. The induction of cycloxygenase by colony stimulating factor-1. Prostaglandins Leukot Essent Fatty Acids 1989;36:101-6.

22 Raines EW, Rosenfeld ME, Ross R. The role of macrophages. In: Fuster V, Ross R, Topol EJ, eds. Atherosclerosis and coronary artery disease. Philadelphia: Lippincott Raven, 1996:539-55.

23 De Gaetano G, Cerletti C, Dejana E, et al. Pharmacology of platelet inhibition in humans: implications of the salicylate-aspirin interaction. Circulation 1985;72:1185-93.

24 Lyberg T, Stanley ER, Prydz H. Colony stimulating factor-1 induces thromboplastin activity in murine macrophages and human monocytes. J Cell Physiol 1987; 132:367-70.

25 Narahara N, Enden T, Wiiger M, et al. Polar expression of tissue factor in human umbilical vein endothelial cells. Arterioscler Thromb 1994; 14:1815-20.

26 Wakefield TW, Greenfield U, Rolfe MW, et al. Inflammatory and procoagulant mediator interactions in an experimental baboon model of venous thrombosis. Thromb Haemost 1993;69:164-72.

27 Topol EJ, Quinn MJ. Common variations in platelet glycoproteins: pharmacogenomic implications. Pharmacogenomics 2001;2:341-52.

28 Neri Serneri GG, Gensini GF, Poggesi L, et al. The role of extra-platelet thromboxane A2 in unstable angina investigated with a dual thromboxane A2 inhibitor: importance of activated monocytes. Coron Artery Dis 1994:5: 137-45

29 Deedwania P, Carbejal EV. Silent ischemia during daily life is an independent predictor of mortality in stable angina. Circulation 1990;81:748-56.

30 Saitoh T, Kishiba H, Tsukada Y, et al. Clinical significance of increased plasma concentration of MCSF in patients with angina pectoris. J Am Coll Cardiol 2000;35:655-65. 